\title{
Pre-transplant histological assessment provides a useful predictor of subsequent kidney allograft function
}

\section{AB Brayne ${ }^{1}$, PB Trotter ${ }^{2}$, DL Hart ${ }^{1}$, GJ Pettigrew², MR Clatworthy ${ }^{1}$}

1 Department of Medicine, University of Cambridge 2 Department of Surgery, University of Cambridge

\section{Introduction}

- Organ shortage is a major challenge in transplantation

- Marginal donors are increasingly used

- Remuzzi score (RS) is a histological assessment of renal grafts comprising glomerular, tubular, interstitial and vascular components

- Remuzzi score require validation as a predictive tool

- This retrospective observational study sought to investigate remuzzi scores as a predictor of allograft function

\section{Methods}

- Retrospective observational study at Addenbrookes Hospital, Cambridge

- All time zero and pre-implantation biopsy's of deceased donor kidneys (both DCD and DBD) with an a remuzzi score

- January $1^{\text {st }} 2010-31^{\text {st }}$ August 2016

- Patient outcomes:

- 1 year death censored graft survival

- 1 year eGFR

\section{Results}

- 495 transplanted deceased donors kidneys with a wide distribution of RS

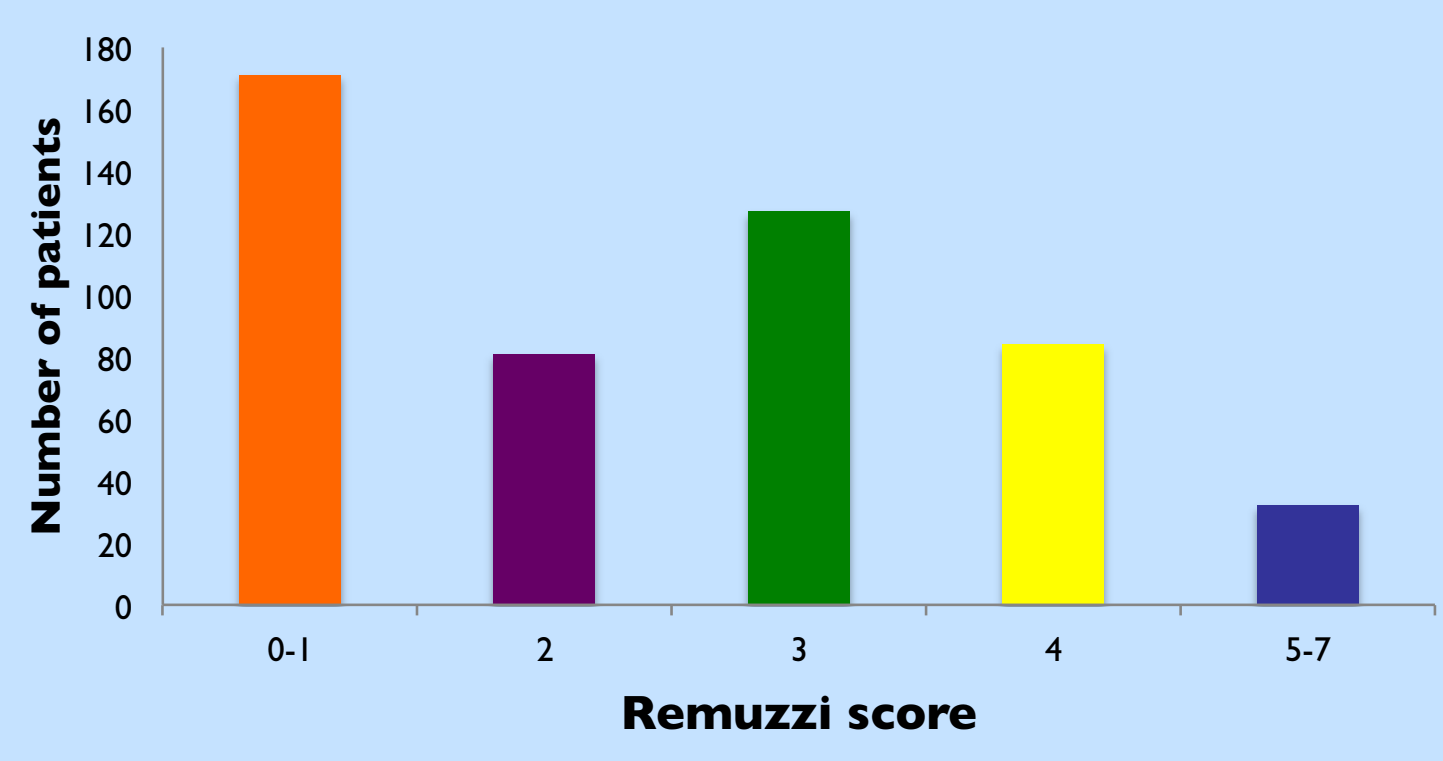

\section{Graft survival}

Unadjusted 5 year death censored graft survival stratified by Remuzzi Score:

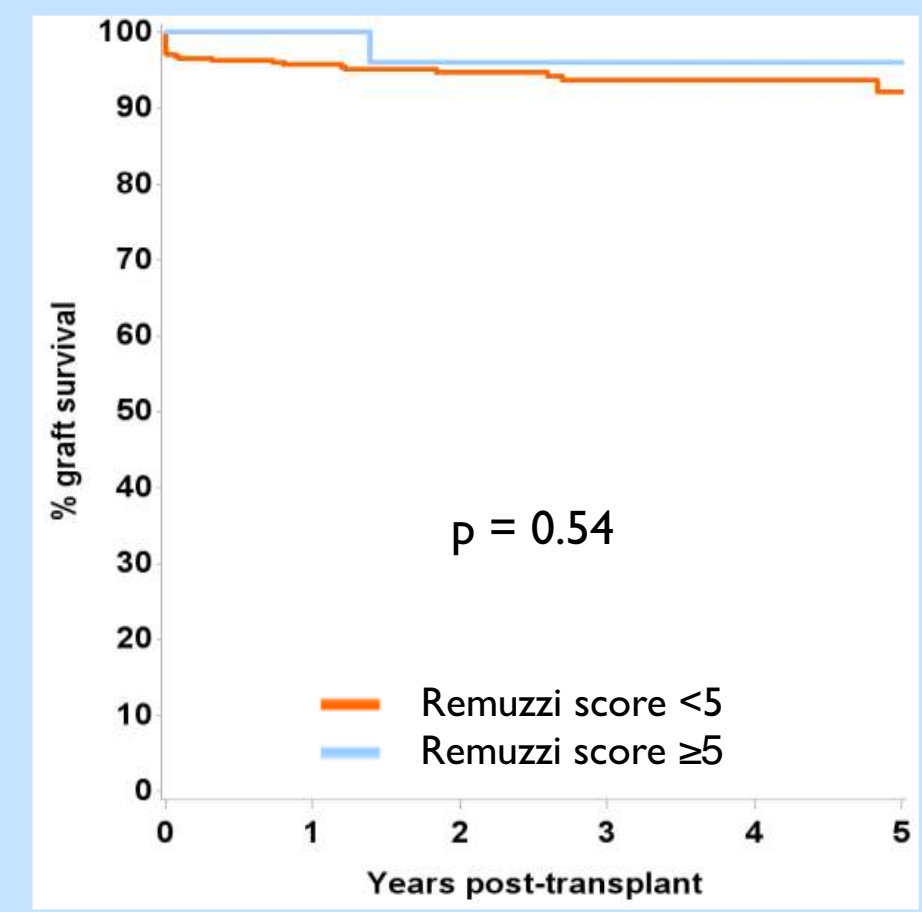

- Unadjusted 1 and 5 year graft survival was similar regardless of the donor kidney

- We speculate that this may be due to masking of long term outcomes due to inclusion of recent transplants.

- There is a reduced rate of primary non function in recipients of kidneys of $R S \geq 5$ compared to our previous study (Mallon et al, AJT 2015)

\section{Graft function}

1 year eGFR was significantly better in those who received a kidney with a lower remuzzi score

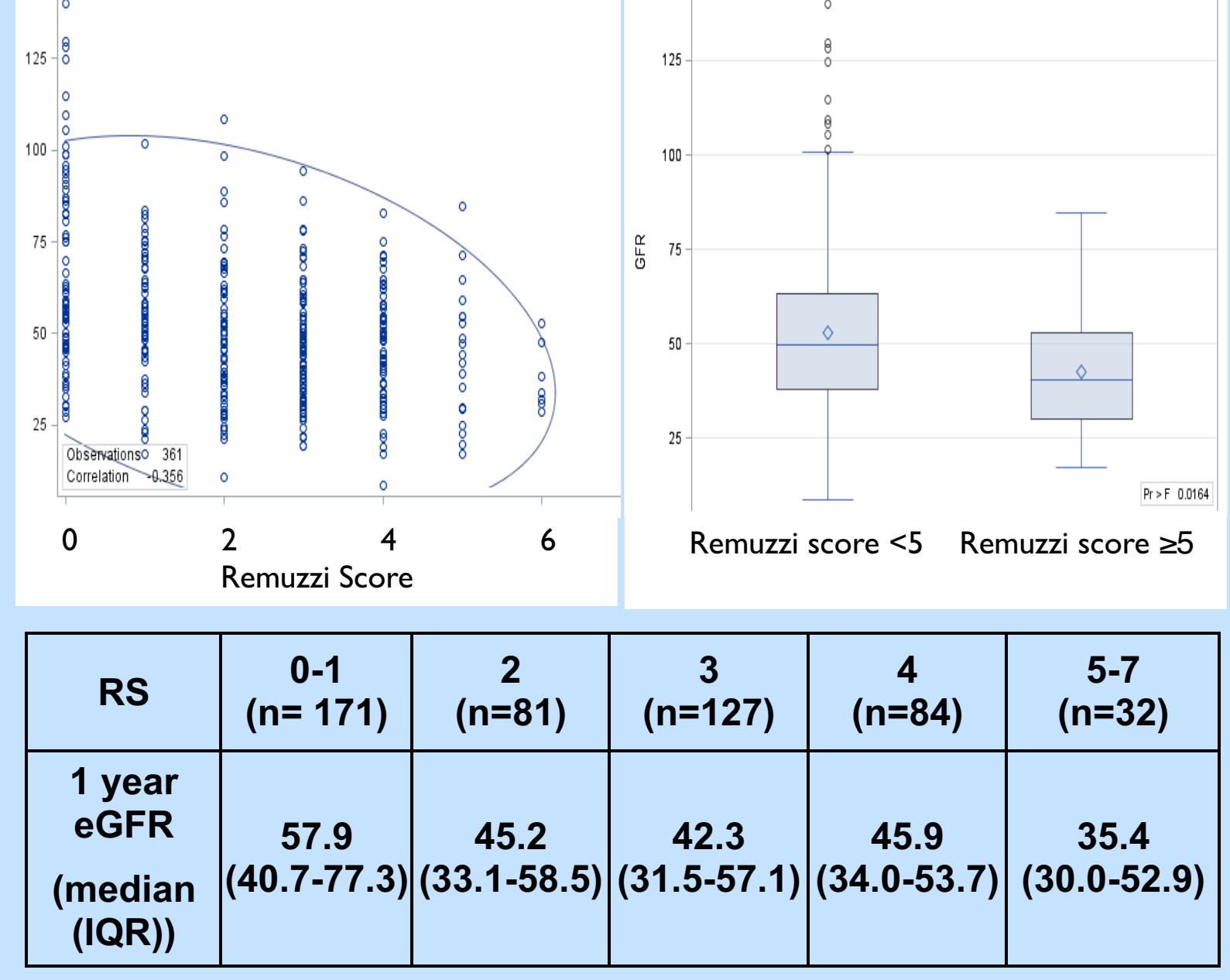

-Left panel - 1 year eGFR by Remuzzi score in single kidney transplant recipients, $\mathrm{p}<0.01$

-Right panel -1 year eGFR grouped RS ( $<4$ and $\geq 5$ ) are significantly different (effect size $12 \mathrm{ml} / \mathrm{min}, p=0.02$ ). One year eGFR of recipients of deceased donor kidneys with a $R S \geq 5$ was worse than those with a RS $<5$

\section{Conclusion}

- Recipients of kidneys with a $R S \geq 5$ had comparable graft survival at one year to those with a $\mathrm{RS}<5$

- $R S$ is effective at predicting one year eGFR in deceased donor renal allografts

- Transplanted single kidneys with a RS of 4 had good graft function at 1 year

- Some kidneys with a score of $>5$ have reasonable outcomes, highlighting the need for additional biomarkers to improve prediction of subsequent function

- A multi-centre randomised clinical trial is required to further validate this approach

Corresponding author Adam Brayne ab988@cam.ac.uk 